\title{
Swelling/Shrinkage Induced by Shear in Narrow Pores
}

\author{
H. Hoang and G. Galliero* \\ Laboratoire des Fluides Complexes et leurs Réservoirs (UMR-5150 with CNRS and \\ TOTAL), Université de Pau et des Pays de l'Adour, BP 1155, 64013 PAU Cedex, \\ France \\ *guillaume.galliero@univ-pau.fr
}

\begin{abstract}
In this work, using molecular dynamics simulations, we have explored the swelling/shrinkage of a simple slit nano-pore immerged in a Lennard-Jones liquid reservoir induced by shear. It is shown that the pore can swell or shrink when the solid walls are displaced in a direction parallel to the fluid-solid interface. This is due to the fact that the normal pressure of the confined fluid appreciably varies with the relative structural ordering between the solid (crystalline) surfaces for a given pore size. Thus, when the solid walls are moved at a constant velocity, the instantaneous pore size oscillates with time and yields, on average, a shear-induced swelling/shrinkage.
\end{abstract}

\section{Introduction}

Understanding the mechanical response of a tight porous medium in which pores are filled partially or completely by a fluid and are subject to an external phenomena, e.g. external stress, plays an important role in many engineering problems: low permeability reservoirs (Shale, Coal seams) in petroleum engineering, activated carbons/zeolites in chemical engineering processes, cement paste in civil engineering, etc. (Coussy 2010, Israelachvili 2010, Karniadakis et al. 2004, Anderson et al. 2010). In such situations where the pore sizes are of the order of one to ten molecular sizes, the main problem is how to take into account the solid-fluid interaction in the mechanical response of the whole porous medium composed of the fluid and the solid matrix (Anderson et al. 2010, Hensen and Smit 2002, Vandamme et al. 2010, Pijaudier-Cabot et al. 2010, Malikova et al. 2010, Brochard et al. 2012).

In a tight porous medium, the surface effects due to the fluid adsorbed on the solid surface and the molecular packing due to confinement can be dominant over the volume effects (Israelachvili 2010, Karniadakis et al. 2004, Schoen 1993, Hansen and McDonald 2006). Among others, this can lead to the swelling of the porous medium (Anderson et al. 2010, Hensen and Smit 2002, Vandamme et al. 2010, PijaudierCabot et al. 2010, Malikova et al. 2010, Brochard et al. 2012). To take into account these effects poromechanics approaches were proposed in the literature. PijaudierCabot et al. (2010) revisited classical poromechanics in the context of microporous materials with a continuous pore size distribution. To account for the surface effects, the constitutive equations were re-formulated by introducing an apparent porosity and an interaction free energy that are related to the Gibbs adsorption isotherm. In a recent study, Brochard et al. (2012) derived poroelastic constitutive equations for a generic 
porous medium, in which the surface effects are characterized by the amount of fluid adsorbed that was shown to depend on both the fluid bulk pressure and the strain of the medium.

It should be noticed that these constitutive equations for microporous medium always assume that the adsorption stress, i.e. the pressure (normal to the solid phase) of the fluid in the pore, sometimes called solvation pressure, and the density of the fluid in the pore are independent on the deviatoric strain. Thus, the volume of a pore is assumed to be unchanged when the porous medium is sheared. As we will show later, this can be inadequate when dealing with some microporous medium in which the solid phase is highly structured and the solid-fluid interactions are strong. This is simply due to the fact that in a slit nanopore configuration, the relative ordering between the solid surfaces has a significant effect on the properties of the fluid in the pore (Schoen 1993, Hoang and Galliero 2013).

Thus, in this work using extensive molecular dynamics (MD) simulations we explore the volumetric deformation of a simple saturated (by a liquid) slit pore induced by the solid displacement in the direction parallel with the solid-fluid interface. To do so, we analyze the swelling/shrinkage (i.e. the gap between the two flat walls) due to a relative displacement of the solid walls, see Fig. 1.

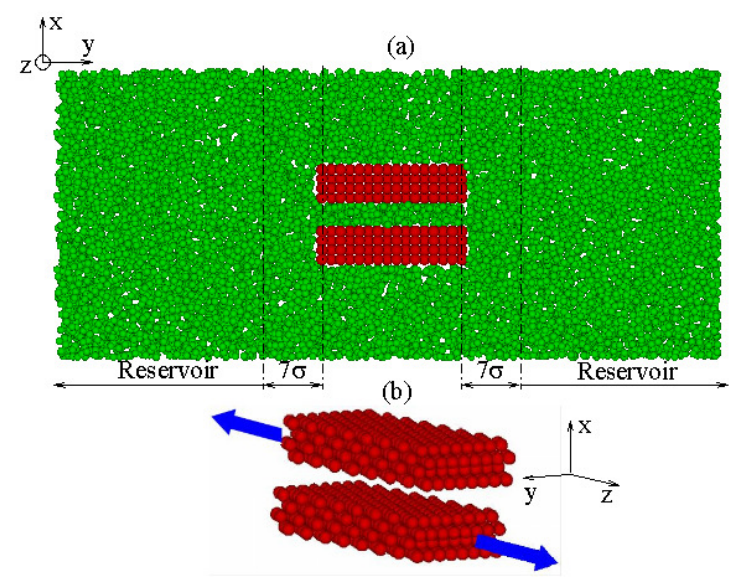

Figure 1: (a): Side view of the simulation cell used in the Grand Canonical like Molecular Dynamics. (b): A schematic representation of the solid displacement in the direction parallel to the solid-fluid interface.

\section{MOLECULAR DYNAMICS SIMULATIONS}

All fluid and solid molecules have been modeled as spherical ones. All interactions are described by a classical truncated Lennard-Jones (LJ) 12-6 potential between pairs of particle:

$$
U_{L J}(r)=\left\{\begin{array}{ccc}
4 \varepsilon\left[\left(\frac{\sigma}{r}\right)^{12}-\left(\frac{\sigma}{r}\right)^{6}\right] & \text { if } & r \leq r_{c} \\
0 & \text { if } & r>r_{c}
\end{array}\right.
$$


where $r$ is the distance between the two particles, $\varepsilon$ is the potential depth, $\sigma$ is the particle diameter, and $r_{c}$ is the cut-off diameter ( $=3.5 \sigma$ in this work). In the following the variables have been expressed in usual LJ dimensionless units, noted with a star as superscript. The solid walls are formed by atoms arranged on a faced centered cubic (FCC) lattice with a lattice size $a^{*}=1.6$. It is assumed that their structures are unchanged during the swelling/shrinkage, i.e. the Biot coefficient in the conventional constitutive equations is equal to one (Biot 1941, Coussy 2004). The walls are separated by a distance $L_{\text {Gap }}^{*}$.

To perform the MD simulations, we have employed the constant density Grand Canonical like Molecular Dynamics method proposed by Hoang and Galliero (2012) which is similar to the one proposed by Gao et al. (1997). It consists in simulating explicitly the fluid in the pore in direct contact with reservoirs maintained at the desired density. For that study we have employed $\rho^{*}=0.7$ and temperature $T^{*}=1$ which corresponds to a liquid state in bulk conditions for the cutoff employed in this work (Galliero and Boned 2008). Figure 1 shows a sketch of the simulation box that contains both fluid and solid particles. Periodic boundary conditions (PBC) have been applied on all three directions. The configuration has been chosen so that the fluid out of the pore space is inappreciably influenced by a small change in the pore studied.

To study the swelling/shrinkage of the pore, the solid walls forming the pore are not allowed to move in the $y$ direction but only in the $x$ direction during the simulations. To model the solid displacement in the direction parallel to the solidfluid interface, the walls are prescriptively moved in the $z$ direction, see Fig. 1 . The equation of motion of the centers of mass of the solid walls in the $x$ direction is governed by the classical equation of motion as:

$$
N_{\mathrm{S}} m_{\mathrm{S}} x_{\mathrm{S}}^{\prime \prime}=\sum_{i=1}^{N_{\mathrm{F}}} \sum_{j=1}^{N_{\mathrm{S}}}-\frac{\partial U_{\mathrm{LJ}}\left(r_{i \mathrm{j}}\right)}{\partial r_{i j}} \frac{x_{i \mathrm{j}}}{r_{i j}}
$$

where $N_{\mathrm{S}}, m_{\mathrm{S}}$ and $x_{\mathrm{s}}$ are the number of solid molecules, the mass of a solid molecule and the coordinate of the center of mass in the $x$ direction for each solid wall respectively. To avoid as much as possible the effects due to the finite size of the two solid walls in the $y$ direction, Eq. (2) is computed using only the solid molecules in the sampling region as described in Hoang and Galliero (2012).

\section{RESULTS and DISCUSSIONS}

\subsection{Swelling/Shrinkage induced by walls displacement}

As mentioned previously, the use of the conventional and recently modified constitutive equations leads to an unchanged pore width when the solid walls are moved in the direction parallel to the solid-fluid interfaces. Thus the shear is not assumed to influence the pressure (normal to the walls) in the fluid. However, in a narrow pore for which its size is comparable to the typical distance of interaction between molecules, the relative structural ordering between the solid walls has a significant effect on the fluid in the pore (Schoen 1993, Hoang and Galliero 2013), in particular on the normal pressure of the confined fluid. This means that a relative 
displacement of the two solid walls can modify the normal pressure of the fluid. Hence, a pore can swell or shrink if the solid walls are translated of a given distance in the direction parallel to the solid-fluid interface. That effect is named "static" swelling/shrinkage in the following.

To explore such static swelling/shrinkage of a pore, we have computed the variation of the pore width with the amplitude of the solid walls displacement $\Delta z^{*}$ starting from an out-of-registry initial configuration of the solid walls. To do so, we have performed MD simulations in which, once the system has reached the equilibrium, the solid walls are moved in opposite direction of a distance $\Delta z^{*} / 2$, see Fig. 1(b). It should be noticed that the change from the initial out-of-registry configuration to an in-registry configuration of the two solid walls, corresponds to a distance $\Delta a^{*} / 2$.
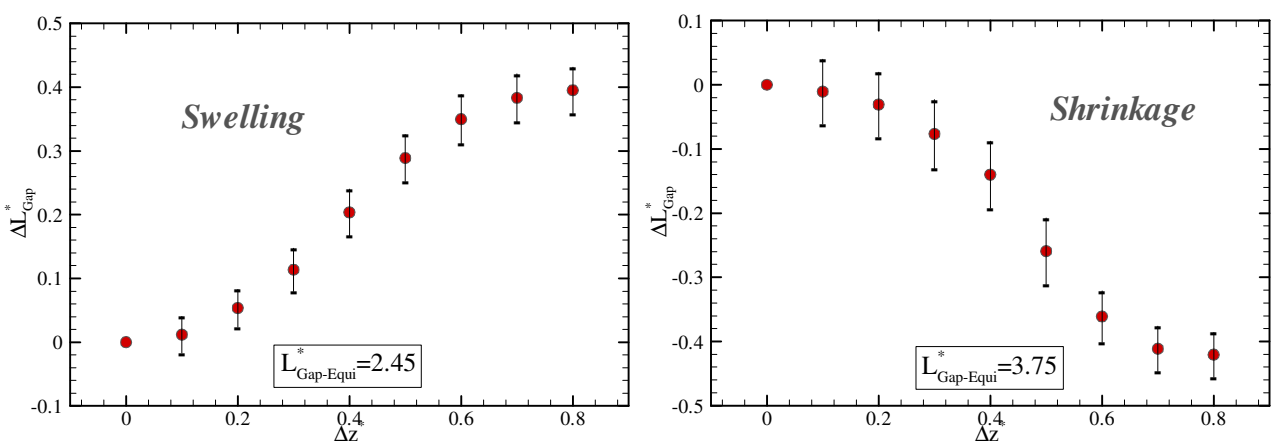

Figure 2: Variation of the pore size for a given solid displacement $\Delta z^{*}$.

Figure 2 depicts the variation in the pore size with the solid displacement $\Delta z^{*}$ for two different initial equilibrium pore widths, $L_{\text {Gap-Equi }}^{*}$. Results clearly indicate that the pore can swell or shrink because of a solid displacement in the direction parallel to the solid-fluid interface. The pore swells when $L_{\text {Gap-Equi }}^{*}=2.45$, whereas it shrinks when $L_{\text {Gap-Equi }}^{*}=3.75$. Such behaviors are simply a consequence of the change in the normal pressure induced by the variation of the relative structural ordering between the solid walls when the solid walls are moved, see Fig. 3 (Hoang and Galliero 2013).

\subsection{Dynamic Swelling/Shrinkage}

To extend the results presented in the previous section, it seems interesting to explore the swelling/shrinkage of a slit pore when the solid walls are moved at a constant velocity, i.e. to induce a dynamic swelling/shrinkage. To do so, we have performed MD simulations in which, once the system has reached the equilibrium, the solid walls are moved at a constant velocity in the direction parallel to the solidsurface interface, see Fig. 1. 

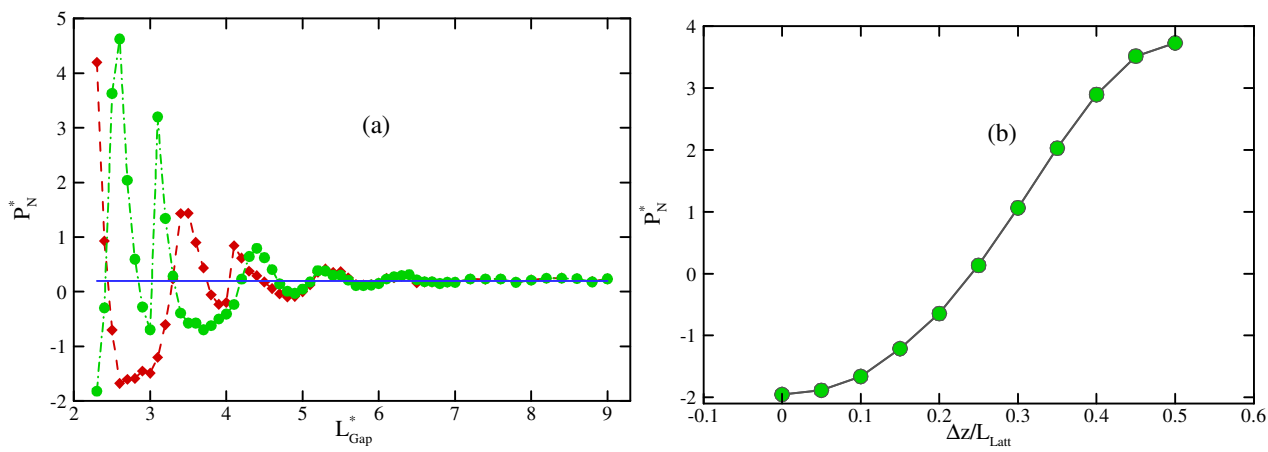

Figure 3: (a) Variation in the normal pressure with gap size. Circles correspond to the in-registry configuration. Diamonds correspond to the out-of-registry configuration.

Solid line represents the pressure in the reservoirs. (b) Variation in the normal pressure with the relative structural ordering between the solid surfaces $\Delta z / L_{\text {Latt }}$ for

$$
L_{\mathrm{Gap}}^{*}=2.65 \text {. }
$$

When the velocity is sufficiently small, it is consistent to assume that the state of the system is completely re-equilibrated at each moment. In other words, the pore size at any time should depend only on the instantaneous relative structural ordering between the solid surfaces. The instantaneous pore size should so vary between the values corresponding to the in-registry and out-of-registry configurations. Thus, we have carried MD simulations for different wall velocities to check this assumption and to explore the behavior of the dynamic swelling/shrinkage for large shearing rate.
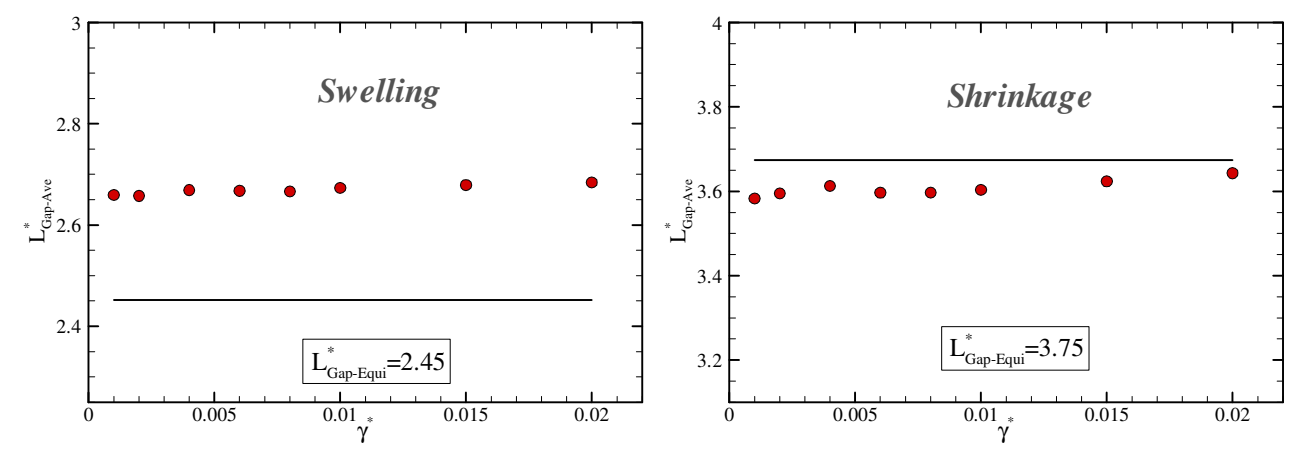

Figure 4: Variation in the average size of the pore with the shear rate $\gamma^{*}=V_{\text {Wall }}^{*} / 2 L_{\text {Gap-Equi }}^{*}$ during dynamic swelling/shrinkage. Symbols correspond to the simulation results. Lines are the size of the pore at equilibrium.

Figure 4 shows the dependence of the average pore size for different shear rates $\gamma^{*}=V_{\text {Wall }}^{*} / 2 L_{\text {Gap-Equi }}^{*}$. Results indicate that the average size is slightly dependent on the shear rate for the range used in this work. More precisely, the pore size is nearly constant below a certain threshold, $\gamma_{c}^{*} \approx 0.01$, and slightly increases with the shear rate when the shear rate is larger than $\gamma_{c}^{*}$. The latter trend is mainly due to the 
fact that the pressure of a fluid usually increases when the wall velocity increases (Hoang and Galliero 2013, Heyes 1986). It should be noticed that $\gamma_{c}^{*}$ is one order of magnitude lower than the shear rate associated to the shear thinning threshold of a bulk LJ liquid (Krögerand and Hess 2000, Galliero and Boned 2009).

Figure 5 displays the variation of the pore size with time for different walls velocities. As expected, all cases exhibit an oscillatory behavior with a period $\theta^{*}=a^{*} / 2 V_{\text {Wall }}^{*}$. This behavior is simply due to the periodic structure of the solid surfaces. It is interesting to notice that the values of peaks and valleys are nearly equal to the equilibrium ones corresponding to the in-registry and out-of-registry configurations for low walls velocities, whereas it is not the case for high velocities, see Figs. 3, 4 and 5. In other words, the walls velocity has a noticeable effect on the instantaneous size of a pore, which is not so obvious when looking only to the average size, see Figs. 4 and 5. In particular, the amplitude of the oscillations is reduced when the walls velocity increases. Such behaviors can be understood in terms of re-equilibration of the fluid in the pore undergoing shear. For low enough shear rate the pore size is governed only by the effect of the relative structural ordering between the solid surfaces, not by the wall velocity. When the shear rate is sufficiently large, the fluid in the pore does not have enough time to re-equilibrate and so the effect of the relative structural ordering is reduced.
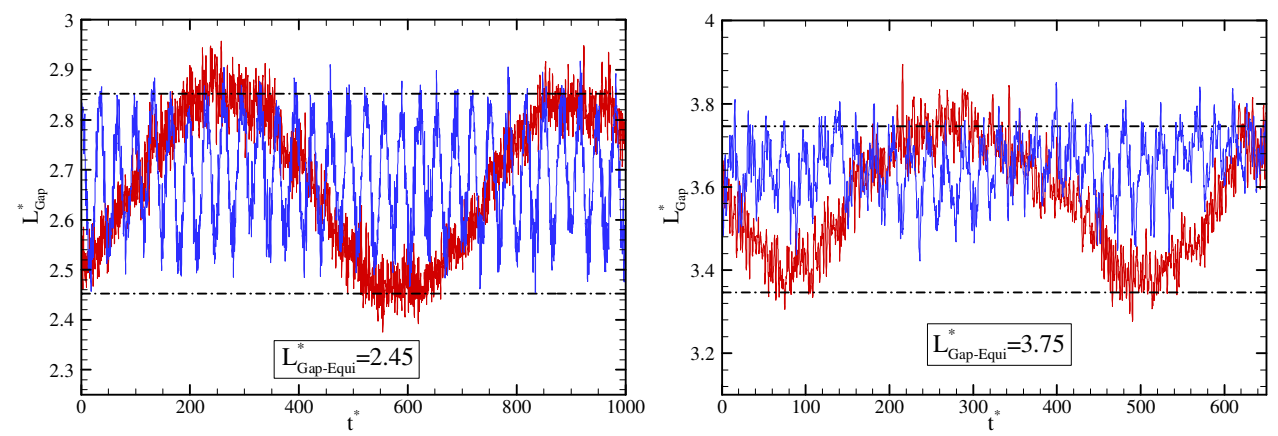

Figure 5: Variation in the pore size with time when the solid walls are moved at a constant velocity. Red curves correspond to $V_{\text {Wall }}^{*} / 2 L_{\text {Gap-Equi }}^{*}=0.001$, blue curves to $V_{\text {Wall }}^{*} / 2 L_{\text {Gap-Equi }}^{*}=0.02$. Dashed-Dotted lines represent the pore size corresponding to the in-registry and out-of-registry configurations at equilibrium.

\section{CONCLUSIONS}

The poro-mechanics approaches have shown to be valuable to describe the deformation of a porous media partially or fully saturated by a fluid. In its present form, such theory assumes that the volume of a pore is unchanged when the porous medium is sheared. However, this is incorrect at the pore scale for systems in which the fluid is highly structured due to a strong solid-fluid interaction. Thus, in this work, using molecular dynamics simulations, we have explored the volumetric deformation of a simple slit nano-pore induced by shear. 
First, the swelling/shrinkage of a pore induced by a displacement of the solid walls (immerged in a fluid reservoir) of a given distance in the direction parallel to the solid-fluid interface has been investigated. Results have shown that, in narrow pores, the average pore size is changed by such solid displacement, i.e. there is a shear-induced swelling/shrinkage. This effect is simply due to the fact that the normal pressure of the simulated fluid appreciably varies with the relative structural ordering between solid surfaces (going from out of registry to in registry configurations).

Then, we have explored the situation in which solid walls are moved at a constant velocity. It has been found that the shear rate employed has only a slight effect on the average pore size, whereas it has a noticeable effect on the instantaneous pore size. More precisely, while all cases exhibit an oscillatory behavior of the instantaneous pore size, which is simply due to the periodic structure of the solid surfaces, the amplitude of the oscillations is reduced when the walls velocity increases. Such behaviors can be understood in terms of re-equilibration of the fluid in the pore undergoing shear.

\section{Acknowledgments}

This work has been supported by the "Failflow" project which is funded by an advanced grant from the European Research Council. We gratefully acknowledge computational facilities (in link with a "Conseil Regional d'Aquitaine" project) provided by Pau University, by the I2M and the MCIA in Bordeaux University.

\section{References}

Anderson, R. L., Ratcliffe, I., Greenwell, H. C., Williams, P. A., Cliffe, S. and Coveney, P. V. (2010) "Clay swelling - A challenge in the oilfield", Earth-Science Reviews 98, 201.

Brochard, L., Vandamme, M. and Pellenq, R. J. M. (2012) "Poromechanics of microporous media", J. Mech. Phys. Solids 60, 606

Biot, M. A. (1941) "General Theory of Three Dimensional Consolidation”, J. Appl. Phys. 12, 155

Coussy, O. (2004) "Poromechanics", John Wiley \& Sons, Chichester

Coussy, O. (2010) "Mechanics and Physics of Porous Solids", John Wiley \& Sons, Chichester

Gao, J., Luedtke, W. D. and Landman, U. (1997) "Structure and solvation forces in confined films: Linear and branched alkanes", J. Chem. Phys. 106, 4309

Galliero, G. and Boned, C. (2009) "Shear viscosity of the Lennard-Jones chain fluid in its gaseous, supercritical, and liquid states", Phys. Rev. E 79, 021201

Galliero, G. and Boned, C. (2008) "Molecular dynamics study of the repulsive form influence of the interaction potential on structural, thermodynamic, interfacial, and transport properties", J. Chem. Phys. 129, 074506

Heyes, D. M. (1986) "Shear thinning and thickening of the Lennard-Jones liquid. A molecular dynamics study", J. Chem. SOC., Faraday Trans. 2, 82, 1365

Hensen, E. J. M. and Smit, B. (2002) "Why Clays Swell" J. Phys. Chem. B 106, 12664 
Hansen, J. and McDonald, I. R. (2006) "Theory of simple liquid", Third Edition, Elsevier

Hoang, H. and Galliero, G. (2012) "Grand canonical-like molecular dynamics simulations: Application to anisotropic mass diffusion in a nanoporous medium", J. Chem. Phys. 136, 184702

Hoang, H. and Galliero, G. (2013) "Shear behavior of a confined thin film: Influence of the molecular dynamics scheme employed" J. Chem. Phys. 138, 054707

Karniadakis, G., Beskok A. and Aluru, N. (2004) "Microflows and Nanoflows", Springer

Kröger, M. and Hess, S. (2000) "Rheological Evidence for a Dynamical Crossover in Polymer Melts via Nonequilibrium Molecular Dynamics”, Phys. Rev. Lett. 85, 1128

Israelachvili, J. (2010) "Intermolecular and Surface Forces", Academic Press, Third Edition

Malikova, N., Dubois, E., Marry, V., Rotenberg, B. and Turq, P. (2010) "Dynamics in Clays - Combining Neutron Scattering and Microscopic Simulation", Zeitschrift für Physikalische Chemie 224, 153

Pijaudier-Cabot, G., Vermorel, R., Miqueu, C. and Mendiboure, B. (2010) "Revisiting poromechanics in the context of microporous materials", C. R. Mecanique 339, 770

Schoen, M. (1993) "Computer simulation of condensed phases in complex geometries", New series m: monographs, Lecture note in physics, $\mathrm{m} 17$

Vandamme, M., Brochard, L., Lecampion B. and Coussy, O. (2010) "Adsorption and strain: The CO2-induced swelling of coal”, J. Mech. Phys. Solids 58, 1489 